\title{
Interval Estimation of Stress-Strength Reliability Based on Lower Record Values from Inverse Rayleigh Distribution
}

\author{
Bahman Tarvirdizade and Hossein Kazemzadeh Garehchobogh \\ Department of Statistics, Islamic Azad University, Maku Branch, Maku, Iran \\ Correspondence should be addressed to Bahman Tarvirdizade; bahtary@gmail.com
}

Received 27 July 2014; Revised 24 October 2014; Accepted 29 October 2014; Published 16 November 2014

Academic Editor: Adiel Teixeira de Almeida

Copyright (c) 2014 B. Tarvirdizade and H. Kazemzadeh Garehchobogh. This is an open access article distributed under the Creative Commons Attribution License, which permits unrestricted use, distribution, and reproduction in any medium, provided the original work is properly cited.

\begin{abstract}
We consider the estimation of stress-strength reliability based on lower record values when $X$ and $Y$ are independently but not identically inverse Rayleigh distributed random variables. The maximum likelihood, Bayes, and empirical Bayes estimators of $R$ are obtained and their properties are studied. Confidence intervals, exact and approximate, as well as the Bayesian credible sets for $R$ are obtained. A real example is presented in order to illustrate the inferences discussed in the previous sections. A simulation study is conducted to investigate and compare the performance of the intervals presented in this paper and some bootstrap intervals.
\end{abstract}

\section{Introduction}

The inverse Rayleigh distribution is an important lifetime distribution in survival analysis that has many applications in the area of reliability studies. Voda [1] presented some properties of the maximum likelihood estimator for inverse Rayleigh distribution and mentioned that the distribution of lifetimes of several types of experimental units can be approximated by the inverse Rayleigh distribution. The cumulative distribution function (cdf) of the inverse Rayleigh distribution with scale parameter $\lambda$ is given by

$$
F(x)=\exp \left(-\frac{\lambda}{x^{2}}\right), \quad x>0, \lambda>0 .
$$

The probability density function (pdf) corresponding to (1) is given by

$$
f(x)=\frac{2 \lambda}{x^{3}} \exp \left(-\frac{\lambda}{x^{2}}\right), \quad x>0, \lambda>0 .
$$

We will denote inverse Rayleigh distribution with scale parameter $\lambda$ by $\operatorname{IR}(\lambda)$.

In many real life applications such as meteorology, hydrology, sports, life-tests and so on, we are dealing with record values. In industry and reliability studies, many products may fail under stress. For example, a wooden beam breaks when sufficient perpendicular force is applied to it, an electronic component ceases to function in an environment of too high temperature, and a battery dies under the stress of time. But the precise breaking stress or failure point varies even among identical items. Hence, in such experiments, measurements may be made sequentially and only values larger (or smaller) than all previous ones are recorded. Data of this type are called record data. Thus, the number of measurements made is considerably smaller than the complete sample size. This measurement saving can be important when the measurements of these experiments are costly if the entire sample was destroyed.

Let $\left\{X_{i}, i \geq 1\right\}$ be a sequence of independent and identically distributed (iid) random variables with an absolutely continuous cumulative distribution function (cdf) $F(x)$ and probability density function (pdf) $f(x)$. An observation $X_{j}$ is called an upper record if its value exceeds all previous observations; that is, $X_{j}$ is an upper record if $X_{j}>X_{i}$ for every $i<j$. An analogous definition can be given for lower records. Records were first introduced and studied by Chandler [2]. Interested readers may refer to Arnold et al. [3], Ahmadi [4], and Gulati and Padgett [5] for more details and applications of record values.

The problem of estimating $R=\operatorname{Pr}(X>Y)$ arises in the context of mechanical reliability of a system with strength $X$ and stress $Y$ and $R$ is chosen as a measure of system reliability. 
The system fails if and only if, at any time, the applied stress is greater than its strength. This type of reliability model is known as the stress-strength model. This problem also arises in situations where $X$ and $Y$ represent lifetimes of two devices and one wants to estimate the probability that one fails before the other. For example, in biometrical studies, the random variable $X$ may represent the remaining lifetime of a patient treated with a certain drug while $Y$ represent the remaining lifetime when treated by another drug. The estimation of stress-strength reliability is very common in the statistical literature. The reader is referred to Kotz et al. [6] for other applications and motivations for the study of the stressstrength reliability.

The problem of estimating the stress-strength reliability $\operatorname{Pr}(X>Y)$ in the inverse Rayleigh distribution was considered by Rao et al. [7] for ordinary samples. Soliman et al. [8] discussed different methods of estimation for the inverse Rayleigh distribution based on lower record values. Sindhu et al. [9] and Feroze and Aslam [10] considered the Bayesian estimation for the parameter of the inverse Rayleigh distribution under left censored data and under singly and doubly type II censored data, respectively. In this paper, we consider the problem of estimating the stress-strength reliability $\operatorname{Pr}(X>Y)$ in the inverse Rayleigh distribution based on lower record values.

The rest of the paper is organized as follows. In Section 2, we discussed likelihood inference for the stress-strength reliability, while in Section 3 we considered Bayesian inference. In Section 4, we presented a real example. A simulation study is described in Section 5. Finally conclusion of the paper is provided in Section 6.

\section{Likelihood Inference}

Let $X \sim \operatorname{IR}\left(\lambda_{1}\right)$ and $Y \sim \operatorname{IR}\left(\lambda_{2}\right)$ be independent random variables. Let $R=\operatorname{Pr}(X>Y)$ be the stress-strength reliability. Then,

$$
\begin{aligned}
R & =\operatorname{Pr}(X>Y) \\
& =\int_{0}^{\infty} \int_{0}^{x} \frac{2 \lambda_{1}}{x^{3}} \exp \left(-\frac{\lambda_{1}}{x^{2}}\right) \frac{2 \lambda_{2}}{y^{3}} \exp \left(-\frac{\lambda_{2}}{y^{2}}\right) d y d x \\
& =\frac{\lambda_{1}}{\lambda_{1}+\lambda_{2}} .
\end{aligned}
$$

We will consider estimating $R$ based on lower record values on both variables. Let $\underset{\sim}{r}=\left(r_{1}, \ldots, r_{n}\right)$ be a set of lower records from $\operatorname{IR}\left(\lambda_{1}\right)$ and let $\underset{\sim}{s}=\left(s_{1}, \ldots, s_{m}\right)$ be an independent set of lower records from $\operatorname{IR}\left(\lambda_{2}\right)$. The likelihood functions are given by (Ahsanullah [11])

$$
\begin{array}{ll}
L\left(\lambda_{1} \mid \underset{\sim}{r}\right)=f\left(r_{n}\right) \prod_{i=1}^{n-1}\left(\frac{f\left(r_{i}\right)}{F\left(r_{i}\right)}\right), & 0<r_{n}<\cdots<r_{1}<\infty, \\
L\left(\lambda_{2} \mid \underline{s}\right)=g\left(s_{m}\right) \prod_{i=1}^{m-1}\left(\frac{g\left(r_{i}\right)}{G\left(r_{i}\right)}\right), & 0<s_{m}<\cdots<s_{1}<\infty,
\end{array}
$$

where $f$ and $F$ are the pdf and cdf of $X \sim \operatorname{IR}\left(\lambda_{1}\right)$, respectively, and $g$ and $G$ are the pdf and $c d f$ of $Y \sim \operatorname{IR}\left(\lambda_{2}\right)$, respectively. Substituting $f, F, g$, and $G$ in the likelihood functions and using (4), we obtain

$$
\begin{aligned}
& L\left(\lambda_{1} \mid \underset{\sim}{r}\right)=\left(2 \lambda_{1}\right)^{n} \exp \left(-\frac{\lambda_{1}}{r_{n}^{2}}\right) \prod_{i=1}^{n}\left(\frac{1}{r_{i}^{3}}\right), \\
& L\left(\lambda_{2} \mid \underset{\sim}{s}\right)=\left(2 \lambda_{2}\right)^{m} \exp \left(-\frac{\lambda_{2}}{s_{m}^{2}}\right) \prod_{i=1}^{m}\left(\frac{1}{s_{i}^{3}}\right) .
\end{aligned}
$$

It can be easily shown that the log-likelihood functions are given by

$$
\begin{aligned}
& \ell\left(\lambda_{1} \mid \underset{\sim}{r}\right)=n \ln \left(2 \lambda_{1}\right)-\frac{\lambda_{1}}{r_{n}^{2}}-\sum_{i=1}^{n} \ln r_{i}^{3}, \\
& \ell\left(\lambda_{2} \mid \underset{\sim}{s}\right)=m \ln \left(2 \lambda_{2}\right)-\frac{\lambda_{2}}{s_{m}^{2}}-\sum_{i=1}^{m} \ln s_{i}^{3} .
\end{aligned}
$$

Differentiate above equations with respect to the parameters $\lambda_{1}$ and $\lambda_{2}$ and equating with zero, the maximum likelihood estimators (MLEs) of $\lambda_{1}$ and $\lambda_{2}$ based on the lower record values are given by

$$
\hat{\lambda}_{1}=n r_{n}^{2}, \quad \hat{\lambda}_{2}=m s_{m}^{2}
$$

Therefore using the invariance properties of the maximum likelihood estimation, the MLE of $R$ is given by

$$
\widehat{R}=\frac{\widehat{\lambda}_{1}}{\widehat{\lambda}_{1}+\widehat{\lambda}_{2}} \text {. }
$$

To study the distribution of $\widehat{R}$ we need the distributions of $\hat{\lambda}_{1}$ and $\hat{\lambda}_{2}$. Consider first $\hat{\lambda}_{1}=n r_{n}^{2}$; the pdf of the $n$th lower record value $R_{n}$ is given by (Ahsanullah [11])

$$
\begin{aligned}
f_{R_{n}}\left(r_{n}\right) & =\frac{1}{(n-1) !} f\left(r_{n}\right)\left[-\ln F\left(r_{n}\right)\right]^{n-1} \\
& =\frac{2}{(n-1) ! r_{n}}\left(\frac{\lambda_{1}}{r_{n}^{2}}\right)^{n} \exp \left(-\frac{\lambda_{1}}{r_{n}^{2}}\right), \quad 0<r_{n}<\infty .
\end{aligned}
$$

Consequently, using standard procedure of transformation of random variables, the pdf of $Z_{1}=\widehat{\lambda}_{1}$ is given by

$$
f_{Z_{1}}\left(z_{1}\right)=\frac{\left(n \lambda_{1}\right)^{n}}{(n-1) ! z_{1}^{n+1}} \exp \left(-\frac{n \lambda_{1}}{z_{1}}\right), \quad z_{1}>0
$$

This is recognized as the inverted gamma distribution; that is, $Z_{1} \sim \operatorname{IGamma}\left(n, n \lambda_{1}\right)$. Similarly, the pdf of $Z_{2}=\widehat{\lambda}_{2}$ is given by

$$
f_{Z_{2}}\left(z_{2}\right)=\frac{\left(m \lambda_{2}\right)^{m}}{(m-1) ! z_{2}^{m+1}} \exp \left(-\frac{m \lambda_{2}}{z_{2}}\right), \quad z_{2}>0 .
$$


Thus $Z_{2} \sim \operatorname{IGamma}\left(m, m \lambda_{2}\right)$. Therefore we can find the pdf of

$$
\widehat{R}=\frac{\widehat{\lambda}_{1}}{\widehat{\lambda}_{1}+\widehat{\lambda}_{2}}=\frac{Z_{1}}{Z_{1}+Z_{2}}=\frac{1}{1+Z_{2} / Z_{1}} .
$$

Consider $Z_{2} / Z_{1}$. Note that, by the properties of the inverted gamma distribution and its relation with the gamma distribution, we have $\left(n \lambda_{1} / Z_{1}\right) \sim \operatorname{Gamma}(n, 1)$ and $\left(m \lambda_{2} / Z_{2}\right) \sim$ $\operatorname{Gamma}(m, 1)$. Hence $\left(2 n \lambda_{1} / Z_{1}\right) \sim \chi_{2 n}^{2}$ and $\left(2 m \lambda_{2} / Z_{2}\right) \sim$ $\chi_{2 m}^{2}$. Note that, by the independence of two random quantities, we have

$$
\frac{\left(2 n \lambda_{1} / 2 n Z_{1}\right)}{\left(2 m \lambda_{2} / 2 m Z_{2}\right)}=\frac{\lambda_{1} Z_{2}}{\lambda_{2} Z_{1}} \sim F_{(2 n, 2 m)} .
$$

Hence, $\left(Z_{2} / Z_{1}\right)=\left(\lambda_{2} / \lambda_{1}\right) F_{(2 n, 2 m)}$ has a scaled $F$ distribution. It follows that the distribution of $\widehat{R}$ is that of

$$
\frac{1}{1+\left(\lambda_{2} / \lambda_{1}\right) F_{(2 n, 2 m)}}
$$

which can be obtained using simple transformation techniques. This fact can be used to construct the following (1 $\alpha) \%$ confidence interval for $R$ :

$$
\left(\left(1+\frac{z_{2}}{z_{1} F_{\alpha / 2,2 n, 2 m}}\right)^{-1},\left(1+\frac{z_{2}}{z_{1} F_{1-\alpha / 2,2 n, 2 m}}\right)^{-1}\right) .
$$

Records are rare in practice (Arnold et al. [3]) and sample sizes are often very small; however, intervals based on the asymptotic normality of MLEs can be of interest in cases when the number of records is sufficiently large. This is because of their optimal asymptotic properties under very general conditions (Lehmann [12]). Note that $\left(\hat{\lambda}_{1}-\right.$ $\left.\lambda_{1}\right) \rightarrow{ }^{d} N\left(0, I^{-1}\left(\lambda_{1}\right)\right)$ as $n \rightarrow \infty$, where $\left(\rightarrow^{d}\right)$ denotes convergence in distribution and $I^{-1}\left(\lambda_{1}\right)$ is the asymptotic variance given by the reciprocal of the Fisher information:

$$
\left[-E\left(\frac{\partial^{2} \ln L\left(\lambda_{1} \mid \underset{\sim}{r}\right)}{\partial \lambda_{1}^{2}}\right)\right]^{-1}=\frac{\lambda_{1}^{2}}{n} .
$$

Similarly, $\left(\hat{\lambda}_{2}-\lambda_{2}\right) \rightarrow{ }^{d} N\left(0, I^{-1}\left(\lambda_{2}\right)\right)$ as $m \rightarrow \infty$, where $I^{-1}\left(\lambda_{2}\right)=\lambda_{2}^{2} / m$. Let $n \rightarrow \infty$ and $m \rightarrow \infty$ such that $m / n \rightarrow$ $p$ where $0<p<1$; it follows that $\sqrt{n}\left(\widehat{\lambda}_{2}-\lambda_{2}\right) \rightarrow N\left(0, \lambda_{2}^{2} / p\right)$. Since

$$
\begin{aligned}
& R=\frac{\lambda_{1}}{\lambda_{1}+\lambda_{2}}=h\left(\lambda_{1}, \lambda_{2}\right), \\
& \widehat{R}=\frac{\widehat{\lambda}_{1}}{\widehat{\lambda}_{1}+\widehat{\lambda}_{2}}=h\left(\widehat{\lambda}_{1}, \widehat{\lambda}_{2}\right),
\end{aligned}
$$

we have

$$
\begin{aligned}
\sqrt{n}(\widehat{R}-R) & =\sqrt{n}\left(h\left(\widehat{\lambda}_{1}, \hat{\lambda}_{2}\right)-h\left(\lambda_{1}, \lambda_{2}\right)\right) \\
& \longrightarrow{ }^{d} N\left(0, \eta^{2}\right)
\end{aligned}
$$

where

$$
\eta^{2}=\left(\frac{\partial h\left(\lambda_{1}, \lambda_{2}\right)}{\partial \lambda_{1}}\right)^{2} \lambda_{1}^{2}+\left(\frac{\partial h\left(\lambda_{1}, \lambda_{2}\right)}{\partial \lambda_{2}}\right)^{2} \frac{\lambda_{2}^{2}}{p} .
$$

A $(1-\alpha) \%$ approximate confidence interval for $R$ based on this asymptotic result is given by

$$
\left(\widehat{R}-z_{1-\alpha / 2} \frac{\widehat{\eta}}{\sqrt{n}}, \widehat{R}+z_{1-\alpha / 2} \frac{\widehat{\eta}}{\sqrt{n}}\right),
$$

where $\widehat{\eta}$ is obtained by substituting $m / n$ for $p$ and the MLEs $\hat{\lambda}_{1}$ and $\hat{\lambda}_{2}$ in the asymptotic standard deviation $\eta$. In these calculations we assumed that $m$ is the smaller sample in size and $n$ is the larger. However, if this is not the case then the formula for the asymptotic variance in the asymptotic interval should be modified accordingly.

\section{Bayesian Inference}

Consider the likelihood functions of $\lambda_{1}$ and $\lambda_{2}$ based on the two sets of lower record values from the inverse Rayleigh distribution mentioned in previous section. These suggest that the conjugate family of prior distributions for $\lambda_{1}$ and $\lambda_{2}$ is the Gamma family of probability distributions:

$$
\begin{array}{ll}
\pi\left(\lambda_{1}\right)=\frac{\theta_{1}^{\gamma_{1}} \lambda_{1}^{\gamma_{1}-1} e^{-\theta_{1} \lambda_{1}}}{\Gamma\left(\gamma_{1}\right)}, & \lambda_{1}>0, \\
\pi\left(\lambda_{2}\right)=\frac{\theta_{2}^{\gamma_{2}} \lambda_{2}^{\gamma_{2}-1} e^{-\theta_{2} \lambda_{2}}}{\Gamma\left(\gamma_{2}\right)}, & \lambda_{2}>0,
\end{array}
$$

where $\gamma_{1}, \theta_{1}, \gamma_{2}$, and $\theta_{2}$ are the parameters of the prior distributions of $\lambda_{1}$ and $\lambda_{2}$, respectively. Combining the prior distributions in (21), with the likelihood functions in (5), we obtain the posterior distributions of $\lambda_{1}$ and $\lambda_{2}$ as

$$
\begin{aligned}
& \left(\lambda_{1} \mid \underset{\sim}{r}\right) \sim \operatorname{Gamma}\left(n+\gamma_{1}, \theta_{1}+\left(\frac{1}{r_{n}^{2}}\right)\right), \\
& \left(\lambda_{2} \mid \underset{\sim}{s}\right) \sim \operatorname{Gamma}\left(m+\gamma_{2}, \theta_{2}+\left(\frac{1}{s_{m}^{2}}\right)\right) .
\end{aligned}
$$

It follows that

$$
\begin{aligned}
& 2\left(\theta_{1}+\left(\frac{1}{r_{n}^{2}}\right)\right)\left(\lambda_{1} \mid \underset{\sim}{r}\right) \sim \chi_{2\left(n+\gamma_{1}\right)}^{2}, \\
& 2\left(\theta_{2}+\left(\frac{1}{s_{m}^{2}}\right)\right)\left(\lambda_{2} \mid \underset{\sim}{s}\right) \sim \chi_{2\left(m+\gamma_{2}\right)}^{2} .
\end{aligned}
$$

It follows that $\pi(R \mid \underset{\sim}{\sim} \underset{\sim}{s})$ the posterior distribution of $R$ is equal to that of $(1+C W)^{-1}$, where $W \sim F_{2\left(m+\gamma_{2}\right), 2\left(n+\gamma_{1}\right)}$ and

$$
C=\frac{\left(m+\gamma_{2}\right)\left(\theta_{1}+\left(1 / r_{n}^{2}\right)\right)}{\left(n+\gamma_{1}\right)\left(\theta_{2}+\left(1 / s_{m}^{2}\right)\right)}
$$


The Bayes estimator under squared error loss is the mean of this posterior distribution which may be approximated. A Bayesian $(1-\alpha) \%$ confidence interval for $R$ is given by

$$
\begin{gathered}
\left(\left(C F_{1-\alpha / 2,2\left(m+\gamma_{2}\right), 2\left(n+\gamma_{1}\right)}+1\right)^{-1},\right. \\
\left.\left(C F_{\alpha / 2,2\left(m+\gamma_{2}\right), 2\left(n+\gamma_{1}\right)}+1\right)^{-1}\right) .
\end{gathered}
$$

The case of a noninformative prior can be treated similarly. One of the well-known noninformative priors is the Jeffreys prior that say, $\pi\left(\lambda_{1}\right) \propto \sqrt{\left|I\left(\lambda_{1}\right)\right|}$. This suggests that prior densities for $\lambda_{1}$ and $\lambda_{2}$ are proportional to $1 / \lambda_{1}$ and $1 / \lambda_{2}$, respectively. Using direct arguments one can show that $\left(\lambda_{1}\right.$ | $\underset{\sim}{r}) \sim \operatorname{Gamma}\left(n, 1 / r_{n}^{2}\right)$ and $\left(\lambda_{2} \mid \underline{s}\right) \sim \operatorname{Gamma}\left(m, 1 / s_{m}^{2}\right)$. It follows that the posterior distribution of $R$ is equal to that of $\left(1+C^{\prime} W\right)^{-1}$, where $W \sim F_{(2 m, 2 n)}$ and $C^{\prime}=m s_{m}^{2} / n r_{n}^{2}$. Therefore a Bayesian $(1-\alpha) \%$ confidence interval for $R$ is given by

$$
\left(\left(C^{\prime} F_{1-\alpha / 2,2 m, 2 n}+1\right)^{-1},\left(C^{\prime} F_{\alpha / 2,2 m, 2 n}+1\right)^{-1}\right) .
$$

Now consider the case when the parameters of prior distributions are themselves unknown. We consider the conjugate prior distributions for $\lambda_{1}$ and $\lambda_{2}$ above when the parameters $\theta_{1}$ and $\theta_{2}$ are unknown. In the empirical Bayes model, we must estimate them. So we calculate the marginal distribution of lower records, with densities

$$
\begin{array}{r}
m\left(\underset{\sim}{r} \mid \theta_{1}\right)=\int f_{\sim}^{f_{R}}\left(\underset{\sim}{r} \mid \lambda_{1}\right) \pi\left(\lambda_{1} \mid \theta_{1}\right) d \lambda_{1}, \\
0<r_{n}<\cdots<r_{1}<\infty, \\
m\left(\underset{\sim}{s} \mid \theta_{2}\right)=\int f_{\sim}^{f_{s}}\left(\underset{s}{s} \mid \lambda_{2}\right) \pi\left(\lambda_{2} \mid \theta_{2}\right) d \lambda_{2}, \\
0<s_{m}<\cdots<s_{1}<\infty .
\end{array}
$$

Using (5) and (21), we obtain

$$
\begin{aligned}
& m\left(\underset{r}{\sim} \mid \theta_{1}\right)=\frac{\Gamma\left(n+\gamma_{1}\right) 2^{n} \theta_{1}^{\gamma_{1}}}{\Gamma\left(\gamma_{1}\right)\left(\theta_{1}+\left(1 / r_{n}^{2}\right)\right)^{n+\gamma_{1}}} \prod_{i=1}^{n}\left(\frac{1}{r_{i}^{3}}\right), \\
& m\left(\underline{s} \mid \theta_{2}\right)=\frac{\Gamma\left(m+\gamma_{2}\right) 2^{m} \theta_{2}^{\gamma_{2}}}{\Gamma\left(\gamma_{2}\right)\left(\theta_{2}+\left(1 / s_{m}^{2}\right)\right)^{m+\gamma_{2}}} \prod_{i=1}^{m}\left(\frac{1}{s_{i}^{3}}\right) .
\end{aligned}
$$

It can be shown that the maximum likelihood estimators (MLEs) of $\theta_{1}$ and $\theta_{2}$ based on the marginal distributions (28) are

$$
\hat{\theta}_{1}=\frac{\gamma_{1}}{n r_{n}^{2}}, \quad \hat{\theta}_{2}=\frac{\gamma_{2}}{m s_{m}^{2}}
$$

With substitution $\widehat{\theta}_{1}$ and $\widehat{\theta}_{2}$ for $\theta_{1}$ and $\theta_{2}$ in prior distributions and using similar arguments above, one can show that

$$
\begin{gathered}
\left(\lambda_{1} \mid \underset{\sim}{r}, \widehat{\theta}_{1}\right) \sim \operatorname{Gamma}\left(n+\gamma_{1}, \frac{\left(\gamma_{1}+n\right)}{n r_{n}^{2}}\right), \\
\left(\lambda_{2} \mid \underset{s}{s} \widehat{\theta}_{2}\right) \sim \operatorname{Gamma}\left(m+\gamma_{2}, \frac{\left(\gamma_{2}+m\right)}{m s_{m}^{2}}\right) .
\end{gathered}
$$

It follows that

$$
\begin{aligned}
& 2\left(\frac{\left(\gamma_{1}+n\right)}{n r_{n}^{2}}\right)\left(\lambda_{1} \mid \underset{\sim}{r}, \widehat{\theta}_{1}\right) \sim \chi_{2\left(n+\gamma_{1}\right)}, \\
& 2\left(\frac{\left(\gamma_{2}+m\right)}{m s_{m}^{2}}\right)\left(\lambda_{2} \mid \underset{s}{s} \widehat{\theta}_{2}\right) \sim \chi_{2\left(m+\gamma_{2}\right)} .
\end{aligned}
$$

It follows that $\pi\left(R \mid \underset{\sim}{r}, \widehat{\theta}_{1}, \underset{\sim}{s}, \widehat{\theta}_{2}\right)$; the empirical posterior distribution of $R$ is equal to that of $\left(1+C^{\prime} W\right)^{-1}$, where $W \sim F_{2\left(m+\gamma_{2}\right), 2\left(n+\gamma_{1}\right)}$ and $C^{\prime}=m s_{m}^{2} / n r_{n}^{2}$. A Bayesian $(1-\alpha) \%$ confidence interval for $R$ is given by

$$
\begin{gathered}
\left(\left(C^{\prime} F_{1-\alpha / 2,2\left(m+\gamma_{2}\right), 2\left(n+\gamma_{1}\right)}+1\right)^{-1},\right. \\
\left.\left(C^{\prime} F_{\alpha / 2,2\left(m+\gamma_{2}\right), 2\left(n+\gamma_{1}\right)}+1\right)^{-1}\right) .
\end{gathered}
$$

The construction of highest posterior density (HPD) regions requires finding the set $I=\left\{\theta: \pi(\theta \mid \underset{\sim}{\sim}, \underset{\sim}{s}) \geq k_{\alpha}\right\}$, where $k_{\alpha}$ is the largest constant such that $\operatorname{Pr}(\theta \in I) \geq 1-\alpha$. This often requires numerical optimization techniques. Chen and Shao [13] presented a simple Monte Carlo technique to approximate the HPD region.

\section{A Real Example}

In order to illustrate the inferences discussed in the previous sections, in this section, we present a data analysis for two data sets reported by Stone [14]. He reports an experiment in which specimens of solid epoxy electrical-insulation were studied in an accelerated voltage life test. For each of two voltage levels 52.5 and $57.5 \mathrm{kV}, 20$ specimens were tested. Failure times, in minutes, for the insulation specimens are given as follows.

Data Set 1. It belongs to voltage level 52.5: 4690, 740, 1010, 1190, 2450, 1390, 350, 6095, 3000, 1458, 6200, 550, 1690, 745, 1225, 1480, 245, 600, 246, 1805.

Data Set 2. It belongs to voltage level 57.5: 510, 1000, 252, 408, 528, 690, 900, 714, 348, 546, 174, 696, 294, 234, 288, 444, 390, $168,558,288$.

We fit the inverse Rayleigh distribution to the two data sets separately. We used the Kolmogorov-Smirnov (K-S) tests for each data set to fit the inverse Rayleigh model. It is observed that for data sets 1 and 2, the K-S distances are 0.2119 and 0.2280 with the corresponding $P$ values 0.2878 and 0.2496 , respectively. Therefore, it is clear that inverse Rayleigh model fits well to both the data sets. We plot the empirical distribution functions and the fitted distribution functions in Figures 1 and 2. These Figures show that the empirical and fitted models are very close for each data set.

Next, we consider the lower record values from the above observed data as follows:

$$
\begin{aligned}
& \underset{\sim}{r}: 4690,470,350,245, \\
& \underset{\sim}{s}: 510,252,174,168 .
\end{aligned}
$$

Based on the above data, we obtain the MLEs of $\lambda_{1}$ and $\lambda_{2}$ as 240100 and 112896, respectively. Therefore, the MLE of $R$ 


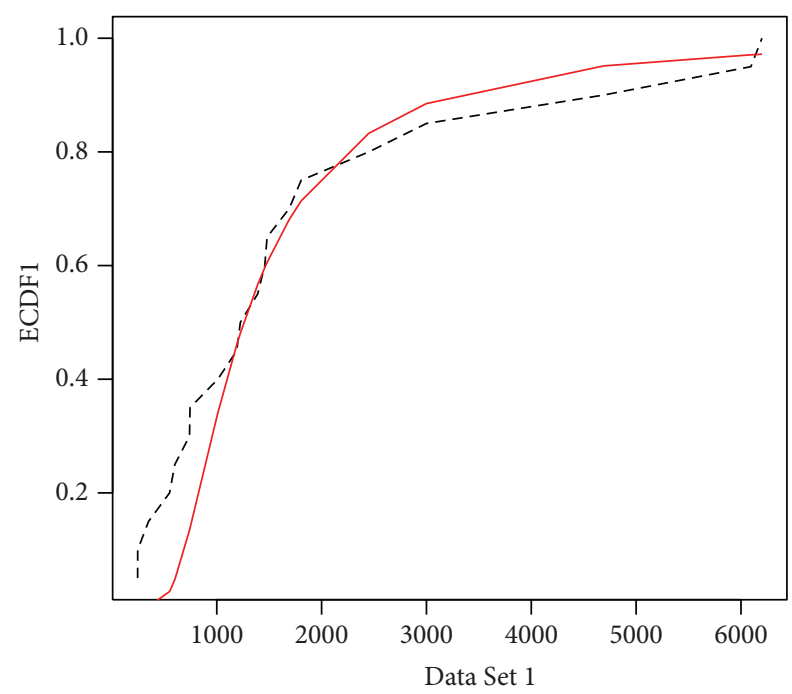

FIgURE 1: The empirical distribution function (dashed) and fitted distribution function for Data Set 1.

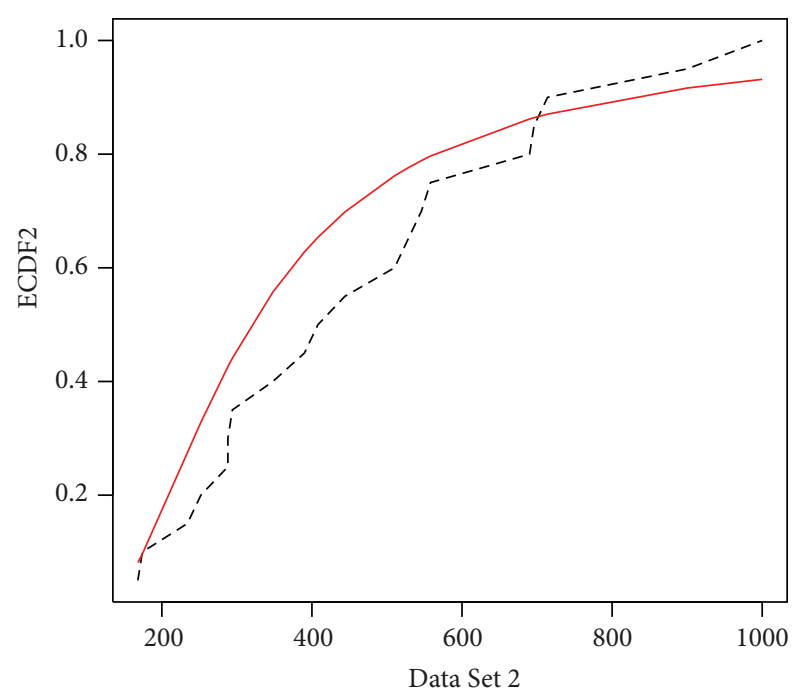

FIgURE 2: The empirical distribution function (dashed) and fitted distribution function for Data Set 2.

becomes $\widehat{R}=0.6802$. The corresponding 95\% confidence interval based on (15) is equal to $(0.3242,0.9041)$. Letting $\gamma_{1}=\gamma_{2}=2, \theta_{1}=3$, and $\theta_{2}=5$, the Bayesian $95 \%$ confidence interval based on (25) is equal to (0.3371, $0.8453)$. So, the Bayesian $95 \%$ confidence interval based on (26) is equal to $(0.3242,0.9041)$. Finally, the Bayesian $95 \%$ confidence interval based on (32) is equal to $(0.3935,0.8745)$.

\section{A Simulation Study}

In this section, a simulation study is conducted to investigate and compare the performance of the confidence intervals presented in this paper and some bootstrap intervals. There are several bootstrap based intervals discussed in the literature (Efron and Tibshirani [15]). It is important here to note that all inference procedures in this paper depend only on the smallest records, $r_{n}$ and $s_{m}$. Therefore we will use the parametric bootstrap based on the marginal distribution of $R_{n}$ as given in (9). In what follows we describe the bootstrapping procedure.

(1) Calculate $\widehat{\lambda}_{1}, \widehat{\lambda}_{2}$, and $\widehat{R}$, the maximum likelihood estimators of $\lambda_{1}, \lambda_{2}$, and $R$ based on $r_{n}$ and $s_{m}$.

(2) Generate $r_{n}^{*}$ from the distribution given in (9) with $\lambda_{1}$ replaced by $\hat{\lambda}_{1}$ and generate $s_{m}^{*}$ similarly.

(3) Calculate $\widehat{\lambda}_{1}^{*}, \widehat{\lambda}_{2}^{*}$, and $\widehat{R}^{*}$ using the $r_{n}^{*}$ and $s_{m}^{*}$ obtained in Step (2).

(4) Repeat Steps (2) and (3) $B$ times to obtain $\widehat{R}_{1}^{*}, \ldots, \widehat{R}_{B}^{*}$. Then we can calculate the following bootstrap intervals.

Normal Interval. The simplest $(1-\alpha)$ bootstrap interval is the normal interval

$$
\left(\widehat{R}-z_{1-\alpha / 2} \widehat{s} e_{\text {boot }}, \widehat{R}+z_{1-\alpha / 2} \widehat{s} e_{\text {boot }}\right),
$$

where $\widehat{s} e_{\text {boot }}$ is the bootstrap estimate of the standard error based on $\widehat{R}_{1}^{*}, \ldots, \widehat{R}_{B}^{*}$.

Basic Pivotal Interval. The $(1-\alpha)$ bootstrap basic pivotal confidence interval is

$$
\left(2 \widehat{R}-\widehat{r}_{(1-\alpha / 2) B}^{*}, 2 \widehat{R}-\widehat{r}_{(\alpha / 2) B}^{*}\right),
$$

where $\widehat{r}_{\beta}^{*}$ is the $\beta$ quantile of $\widehat{R}_{1}^{*}, \ldots, \widehat{R}_{B}^{*}$.

Percentile Interval. The $(1-\alpha)$ bootstrap percentile interval is defined by

$$
\left(\widehat{r}_{(\alpha / 2) B}^{*}, \widehat{r}_{(1-\alpha / 2) B}^{*}\right) ;
$$

that is, just use the $\alpha / 2$ and $1-\alpha / 2$ quantiles of the bootstrap sample.

Interested readers may refer to DiCiccio and Efron [16] and the references contained therein to observe more details.

In the simulation design we used all combinations of $n=5,10,15$ and $m=5,10,15$. We used $\lambda_{1}=1$ and $R=$ $0.1,0.25,0.5$. The value of $\lambda_{2}$ is determined by the choice of $\lambda_{1}$ and $R$. The confidence level taken is $(1-\alpha)=0.90$ and 0.95 . For each combination of the simulation indices we generated 2000 samples of lower records from the distributions of $X$ and $Y$. For each generated pair of samples we calculated the following intervals:

(1) ML: the interval based on the MLE given in (15),

(2) Bayes: the interval based on the Bayes estimator given in (25),

(3) J.B: the interval based on the Bayes estimator given in (26),

(4) E.B: the interval based on the empirical Bayes estimator given in (32),

(5) Norm: the normal interval, 
TABLE 1: Expected lengths and coverage rates (in parentheses) of the confidence intervals with $(1-\alpha)=0.95$.

\begin{tabular}{|c|c|c|c|c|c|c|c|c|c|}
\hline$n$ & $m$ & $R$ & ML & Bayes & J.B & E.B & Norm & Basic & Perc \\
\hline 5 & 5 & 0.1 & $0.277(0.942)$ & $0.406(0.520)$ & $0.272(0.944)$ & $0.190(0.847)$ & $0.284(0.933)$ & $0.276(0.822)$ & $0.276(0.940)$ \\
\hline 5 & 5 & 0.25 & $0.451(0.951)$ & $0.422(0.791)$ & $0.453(0.951)$ & $0.336(0.862)$ & $0.461(0.908)$ & $0.449(0.812)$ & $0.449(0.949)$ \\
\hline 5 & 5 & 0.5 & $0.537(0.947)$ & $0.434(0.988)$ & $0.540(0.956)$ & $0.414(0.868)$ & $0.549(0.886)$ & $0.536(0.798)$ & $0.536(0.941)$ \\
\hline 5 & 10 & 0.1 & $0.220(0.952)$ & $0.339(0.645)$ & $0.217(0.947)$ & $0.172(0.883)$ & $0.257(0.957)$ & $0.250(0.857)$ & $0.250(0.947)$ \\
\hline 5 & 10 & 0.25 & $0.386(0.948)$ & $0.377(0.871)$ & $0.388(0.949)$ & $0.314(0.879)$ & $0.421(0.929)$ & $0.413(0.841)$ & $0.413(0.942)$ \\
\hline 5 & 10 & 0.5 & $0.482(0.956)$ & $0.403(0.976)$ & $0.482(0.949)$ & $0.390(0.883)$ & $0.489(0.903)$ & $0.480(0.833)$ & $0.480(0.937)$ \\
\hline 5 & 15 & 0.1 & $0.203(0.946)$ & $0.300(0.777)$ & $0.202(0.943)$ & $0.164(0.873)$ & $0.248(0.964)$ & $0.241(0.856)$ & $0.241(0.927)$ \\
\hline 5 & 15 & 0.25 & $0.367(0.947)$ & $0.352(0.902)$ & $0.365(0.953)$ & $0.301(0.887)$ & & $0.402(0.866)$ & $0.402(0.933)$ \\
\hline 5 & 15 & 0.5 & $0.460(0.956)$ & $0.386(0.981)$ & $0.459(0.947)$ & $0.375(0.873)$ & $0.466(0.907)$ & $0.458(0.844)$ & $0.458(0.936)$ \\
\hline 10 & 5 & 0.1 & $0.235(0.946)$ & $0.361(0.502)$ & $0.236(0.943)$ & $0.164(0.856)$ & $0.209(0.905)$ & $0.204(0.825)$ & $0.204(0.935)$ \\
\hline 10 & 5 & 0.25 & $0.406(0.961)$ & $0.377(0.721)$ & $0.403(0.935)$ & $0.299(0.846)$ & $0.383(0.911)$ & $0.375(0.833)$ & $0.375(0.945)$ \\
\hline 10 & 5 & 0.5 & $0.482(0.958)$ & & & & & & \\
\hline 10 & 10 & 0.1 & $0.178(0.947)$ & $0.292(0.650)$ & $0.175(0.950)$ & $0.144(0.905)$ & $0.181(0.939)$ & $0.178(0.858)$ & $0.178(0.950)$ \\
\hline 10 & 10 & 0.25 & $0.326(0.958)$ & $0.328(0.828)$ & $0.327(0.955)$ & $0.275(0.913)$ & & $0.326(0.872)$ & $0.326(0.955)$ \\
\hline 10 & 10 & 0.5 & $0.405(0.943)$ & $0.352(0.969)$ & $0.405(0.949)$ & $0.346(0.895)$ & $0.409(0.900)$ & $0.404(0.854)$ & $0.404(0.940)$ \\
\hline 10 & 15 & 0.1 & $0.157(0.955)$ & $0.252(0.735)$ & $0.156(0.952)$ & $0.135(0.913)$ & $0.167(0.952)$ & $0.164(0.875)$ & $0.164(0.950)$ \\
\hline 10 & 15 & 0.25 & $0.297(0.951)$ & $0.300(0.864)$ & $0.296(0.949)$ & $0.258(0.913)$ & $0.307(0.938)$ & $0.304(0.891)$ & $0.304(0.947)$ \\
\hline 10 & 15 & 0.5 & $0.374(0.943)$ & $0.333(0.968)$ & $0.374(0.947)$ & $0.328(0.913)$ & $0.377(0.908)$ & $0.373(0.868)$ & $0.373(0.935)$ \\
\hline 15 & 5 & 0.1 & $0.224(0.946)$ & $0.339(0.490)$ & $0.221(0.935)$ & & & $0.185(0.829)$ & $0.185(0.934)$ \\
\hline 15 & 5 & 0.25 & $0.386(0.951)$ & $0.355(0.706)$ & $0.386(0.947)$ & $0.283(0.871)$ & $0.353(0.894)$ & $0.346(0.835)$ & $0.346(0.931)$ \\
\hline 15 & 5 & 0.5 & $0.459(0.946)$ & $0.367(0.979)$ & $0.459(0.943)$ & $0.354(0.855)$ & $0.463(0.888)$ & $0.455(0.832)$ & $0.455(0.919)$ \\
\hline 15 & 10 & 0.1 & $0.160(0.952)$ & $0.270(0.614)$ & $0.162(0.956)$ & $0.134(0.911)$ & $0.154(0.933)$ & $0.152(0.865)$ & $0.152(0.948)$ \\
\hline 15 & 10 & 0.25 & $0.300(0.943)$ & $0.304(0.776)$ & $0.302(0.950)$ & $0.256(0.909)$ & $0.295(0.911)$ & $0.291(0.869)$ & $0.291(0.937)$ \\
\hline 15 & 10 & 0.5 & $0.374(0.948)$ & $0.327(0.967)$ & $0.375(0.950)$ & $0.323(0.911)$ & $0.378(0.919)$ & $0.374(0.873)$ & $0.374(0.947)$ \\
\hline 15 & 15 & 0.1 & $0.138(0.946)$ & $0.229(0.658)$ & $0.139(0.947)$ & $0.122(0.919)$ & $0.140(0.936)$ & $0.138(0.881)$ & $0.138(0.948)$ \\
\hline 15 & 15 & 0.25 & $0.266(0.953)$ & $0.274(0.847)$ & $0.267(0.951)$ & $0.236(0.920)$ & $0.268(0.929)$ & $0.266(0.884)$ & $0.266(0.950)$ \\
\hline 15 & 15 & 0.5 & $0.339(0.952)$ & $0.305(0.965)$ & $0.339(0.948)$ & $0.303(0.915)$ & $0.342(0.931)$ & $0.339(0.899)$ & $0.339(0.952)$ \\
\hline
\end{tabular}

(6) Basic: the basic pivotal interval,

(7) Perc: the percentile interval.

The empirical coverage probability and expected lengths of intervals are obtained by using the 2000 replications. In the interval based on the Bayes estimators we used $\gamma_{1}=3, \gamma_{2}=5$, $\theta_{1}=2$, and $\theta_{2}=4$ wherever we need them. For bootstrap intervals we used 1000 bootstrap samples. The results of our simulations are given in Tables 1 and 2.

\section{Conclusion and Discussion}

Based on simulation results in Tables 1 and 2, we observe that the length of the intervals is maximized when $R=$ 0.5 and gets shorter and shorter as we move away to the extremes. Increasing the sample size on either variable also results in shorter intervals. The performance of both basic pivotal interval and percentile interval is similar in terms of expected length but in terms of coverage rate percentile interval has the better performance. The percentile interval appears to be the best among bootstrap intervals. The interval based on the MLE and the interval based on the Bayes estimator given in (26) appear to perform almost as well as the percentile interval. The interval based on the Bayes estimator given in (25) has best coverage rate and the short expected length between the other intervals when $R=0.5$, but it has the low coverage rate and the long expected length for small values of $R$ since it is dependent on choice of $\theta_{1}$ and $\theta_{2}$ values. Furthermore, the interval based on the empirical Bayes estimator has the shortest expected length between the other intervals but it has the low coverage rate.

Based on the above discussion, we can conclude that, between the intervals obtained in this paper, the intervals based on the MLE, and the Bayes estimator given in (26) and between the bootstrap intervals, percentile interval simultaneously has the short expected length and very good coverage rate in comparison with the other intervals. Hence, we recommend using this confidence interval in all. 
TABLE 2: Expected lengths and coverage rates (in parentheses) of the confidence intervals with $(1-\alpha)=0.90$.

\begin{tabular}{|c|c|c|c|c|c|c|c|c|c|}
\hline$n$ & $m$ & $R$ & ML & Bayes & J.B & E.B & Norm & Basic & Perc \\
\hline 5 & 5 & 0.1 & $0.223(0.890)$ & $0.343(0.504)$ & $0.224(0.895)$ & $0.159(0.766)$ & $0.235(0.900)$ & $0.223(0.782)$ & $0.223(0.890)$ \\
\hline 5 & 5 & 0.25 & $0.378(0.908)$ & $0.359(0.632)$ & $0.383(0.892)$ & $0.284(0.775)$ & $0.385(0.868)$ & $0.378(0.768)$ & $0.378(0.908)$ \\
\hline 5 & 5 & 0.5 & $0.460(0.897)$ & $0.369(0.961)$ & $0.462(0.912)$ & $0.351(0.784)$ & $0.461(0.832)$ & $0.460(0.746)$ & $0.460(0.895)$ \\
\hline 5 & 10 & 0.1 & $0.180(0.899)$ & $0.288(0.650)$ & $0.183(0.890)$ & $0.145(0.804)$ & $0.213(0.931)$ & $0.200(0.819)$ & $0.200(0.892)$ \\
\hline 5 & 10 & 0.25 & $0.331(0.910)$ & $0.320(0.744)$ & $0.329(0.901)$ & $0.265(0.802)$ & $0.356(0.894)$ & $0.350(0.808)$ & $0.350(0.892)$ \\
\hline 5 & 10 & 0.5 & $0.411(0.897)$ & $0.342(0.951)$ & $0.411(0.904)$ & $0.330(0.799)$ & $0.410(0.846)$ & $0.409(0.778)$ & $0.409(0.893)$ \\
\hline 5 & 15 & 0.1 & $0.171(0.895)$ & $0.255(0.733)$ & $0.171(0.895)$ & $0.139(0.803)$ & $0.209(0.936)$ & $0.197(0.834)$ & $0.197(0.879)$ \\
\hline 5 & 15 & 0.25 & $0.309(0.894)$ & $0.299(0.797)$ & $0.310(0.910)$ & $0.255(0.837)$ & $0.341(0.881)$ & $0.334(0.819)$ & $0.334(0.879)$ \\
\hline 5 & 15 & 0.5 & $0.392(0.900)$ & $0.328(0.938)$ & $0.391(0.902)$ & $0.318(0.812)$ & $0.389(0.845)$ & $0.389(0.793)$ & $0.389(0.883)$ \\
\hline 10 & 5 & 0.1 & $0.190(0.890)$ & $0.305(0.492)$ & $0.191(0.907)$ & $0.135(0.805)$ & $0.175(0.869)$ & $0.169(0.785)$ & $0.169(0.889)$ \\
\hline 10 & 5 & 0.25 & $0.341(0.895)$ & $0.319(0.588)$ & $0.340(0.900)$ & $0.253(0.795)$ & $0.323(0.848)$ & $0.320(0.796)$ & $0.320(0.886)$ \\
\hline 10 & 5 & 0.5 & $0.411(0.900)$ & $0.330(0.950)$ & $0.410(0.899)$ & $0.317(0.792)$ & $0.410(0.852)$ & $0.410(0.786)$ & $0.410(0.892)$ \\
\hline 10 & 10 & 0.1 & $0.145(0.901)$ & $0.246(0.611)$ & $0.145(0.898)$ & $0.120(0.834)$ & $0.150(0.907)$ & $0.145(0.832)$ & $0.145(0.895)$ \\
\hline 10 & 10 & 0.25 & $0.276(0.897)$ & $0.277(0.705)$ & $0.274(0.905)$ & $0.231(0.848)$ & $0.278(0.885)$ & $0.276(0.834)$ & $0.276(0.898)$ \\
\hline 10 & 10 & 0.5 & $0.344(0.897)$ & $0.298(0.934)$ & $0.344(0.904)$ & $0.293(0.835)$ & $0.344(0.862)$ & $0.344(0.825)$ & $0.344(0.898)$ \\
\hline 10 & 15 & 0.1 & $0.129(0.899)$ & $0.212(0.655)$ & $0.130(0.900)$ & $0.112(0.844)$ & $0.139(0.913)$ & $0.134(0.839)$ & $0.134(0.900)$ \\
\hline 10 & 15 & 0.25 & $0.248(0.895)$ & $0.254(0.750)$ & $0.250(0.892)$ & $0.182(0.853)$ & $0.256(0.882)$ & $0.253(0.834)$ & $0.253(0.896)$ \\
\hline 10 & 15 & 0.5 & $0.318(0.903)$ & $0.282(0.915)$ & $0.318(0.896)$ & $0.278(0.850)$ & $0.318(0.876)$ & $0.318(0.847)$ & $0.318(0.904)$ \\
\hline 15 & 5 & 0.1 & $0.182(0.913)$ & $0.286(0.407)$ & $0.179(0.898)$ & $0.125(0.785)$ & $0.159(0.878)$ & $0.155(0.814)$ & $0.155(0.897)$ \\
\hline 15 & 5 & 0.25 & $0.321(0.903)$ & $0.300(0.530)$ & $0.323(0.889)$ & $0.238(0.763)$ & $0.295(0.850)$ & $0.293(0.800)$ & $0.293(0.884)$ \\
\hline 15 & 5 & 0.5 & $0.391(0.893)$ & $0.311(0.951)$ & $0.390(0.884)$ & $0.299(0.773)$ & $0.390(0.841)$ & $0.389(0.789)$ & $0.389(0.878)$ \\
\hline 15 & 10 & 0.1 & $0.133(0.899)$ & $0.227(0.501)$ & $0.134(0.894)$ & $0.111(0.836)$ & $0.131(0.897)$ & $0.128(0.844)$ & $0.128(0.896)$ \\
\hline 15 & 10 & 0.25 & $0.251(0.881)$ & $0.255(0.674)$ & $0.250(0.891)$ & $0.212(0.831)$ & $0.247(0.865)$ & $0.245(0.827)$ & $0.245(0.888)$ \\
\hline 15 & 10 & 0.5 & $0.317(0.896)$ & $0.277(0.935)$ & $0.318(0.907)$ & $0.273(0.852)$ & $0.317(0.862)$ & $0.317(0.820)$ & $0.317(0.897)$ \\
\hline 15 & 15 & 0.1 & $0.115(0.899)$ & $0.193(0.620)$ & $0.116(0.904)$ & $0.102(0.858)$ & $0.118(0.913)$ & $0.115(0.859)$ & $0.115(0.898)$ \\
\hline 15 & 15 & 0.25 & $0.225(0.906)$ & $0.231(0.738)$ & $0.224(0.900)$ & $0.199(0.852)$ & $0.226(0.890)$ & $0.225(0.858)$ & $0.225(0.905)$ \\
\hline 15 & 15 & 0.5 & $0.287(0.901)$ & $0.258(0.927)$ & $0.287(0.905)$ & $0.256(0.871)$ & $0.286(0.872)$ & $0.287(0.840)$ & $0.287(0.898)$ \\
\hline
\end{tabular}

\section{Conflict of Interests}

The authors declare that there is no conflict of interests regarding the publication of this paper.

\section{Acknowledgments}

The authors express their sincere thanks to the editor and the referees for their constructive criticisms and excellent suggestions which led to a considerable improvement in the presentation of the paper.

\section{References}

[1] R. G. Voda, "On the inverse Rayleigh variable," Reports of Statistical Application Researcho f the Union of Japanese Scientists and Engineers, vol. 19, no. 4, pp. 15-21, 1972.

[2] K. N. Chandler, "The distribution and frequency of record values," Journal of the Royal Statistical Society B, vol. 14, pp. $220-$ 228, 1952.

[3] C. B. Arnold, N. Balakrishnan, and H. N. Nagaraja, Records, Wiley, 1998.

[4] J. Ahmadi, Record values, theory and applications [Ph.D. thesis], Ferdowsi University of Mashhad, Mashhad, Iran, 2000.
[5] S. Gulati and W. J. Padgett, Parametric and Nonparametric Inference from Record-Breaking Data, vol. 172 of Lecture Notes in Statistics, Springer, New York, NY, USA, 2003.

[6] S. Kotz, Y. Lumelskii, and M. Pensky, The Stress-strength Model and its Generalizations: Theory and Applications, World Scientific Publishers, 2003.

[7] G. S. Rao, R. R. L. Kantam, K. Rosaiah, and J. P. Reddy, "Estimation of stress-strength reliability from inverse Rayleigh distribution," Journal of Industrial and Production Engineering, vol. 30, no. 4, pp. 256-263, 2013.

[8] A. Soliman, A. E. Amin, and A. A. Abd-El Aziz, "Estimation and prediction from inverse Rayleigh distribution based on lower record values," Applied Mathematical Sciences, vol. 4, no. 62, pp. 3057-3066, 2010.

[9] T. N. Sindhu, M. Aslam, and N. Feroze, "Bayes estimation of the parameters of the inverse Rayleigh distribution for left censored data," ProbStat Forum, vol. 6, pp. 42-59, 2013.

[10] N. Feroze and M. Aslam, "On posterior analysis of inverse Rayleigh distribution under singly and doubly type II censored data," International Journal of Probability and Statistics, vol. 1, no. 5, pp. 145-152, 2012.

[11] M. Ahsanullah, Record Values, Theory and Applications, University Press of America, Lanham, Md, USA, 2004.

[12] E. L. Lehmann, Elements of Large Sample Theory, Springer, New York, NY, USA, 1999. 
[13] M.-H. Chen and Q.-M. Shao, "Monte Carlo estimation of Bayesian credible and HPD intervals," Journal of Computational and Graphical Statistics, vol. 8, no. 1, pp. 69-92, 1999.

[14] G. C. Stone, Statistical analysis of accelerated aging tests on solid electrical insulation [M.S. thesis], University of Waterloo, Waterloo, Ont, Canada, 1978.

[15] B. Efron and R. J. Tibshirani, An Introduction to the Bootstrap, Chapman \& Hall, New York, NY, USA, 1993.

[16] T. J. DiCiccio and B. Efron, "Bootstrap confidence intervals," Statistical Science, vol. 11, no. 3, pp. 189-228, 1996. 

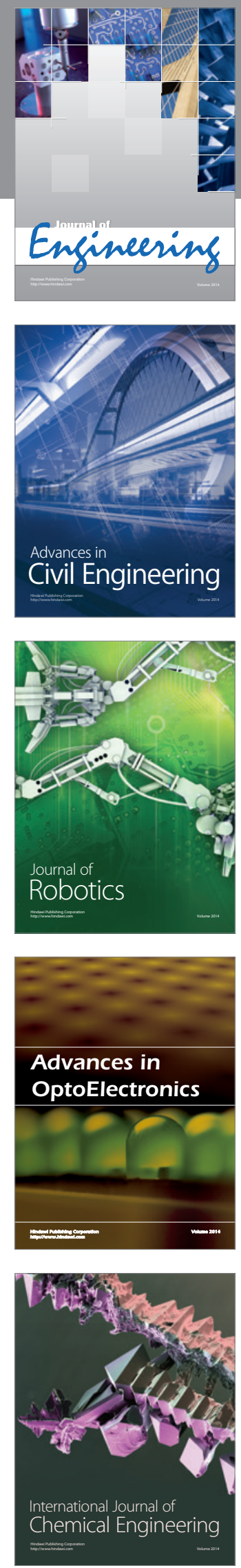

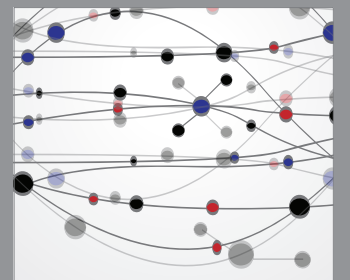

The Scientific World Journal
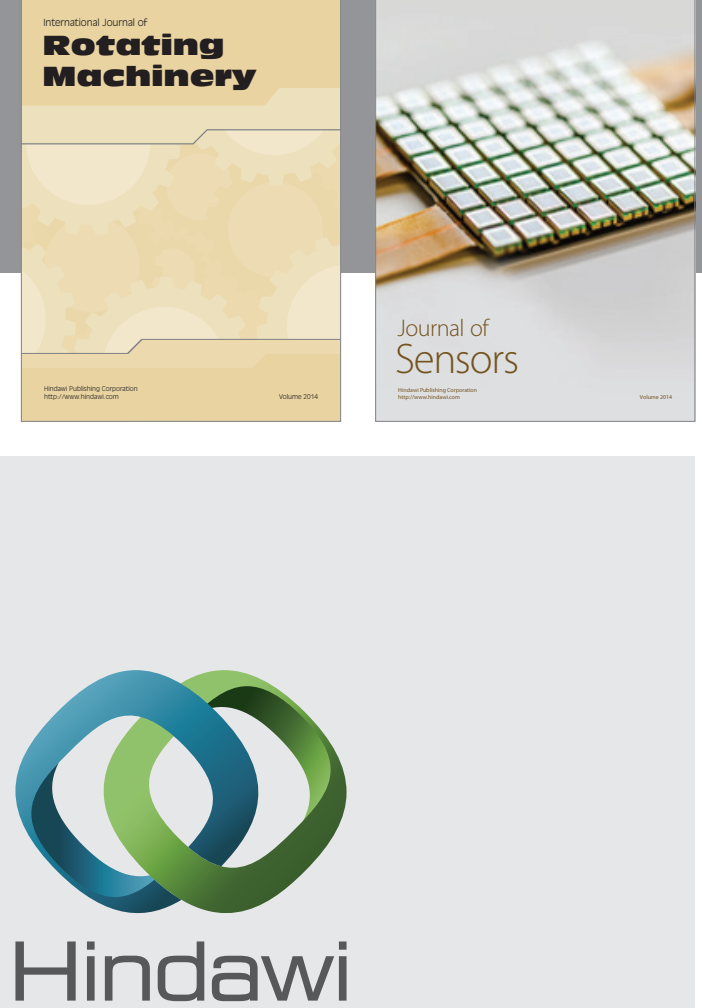

Submit your manuscripts at http://www.hindawi.com
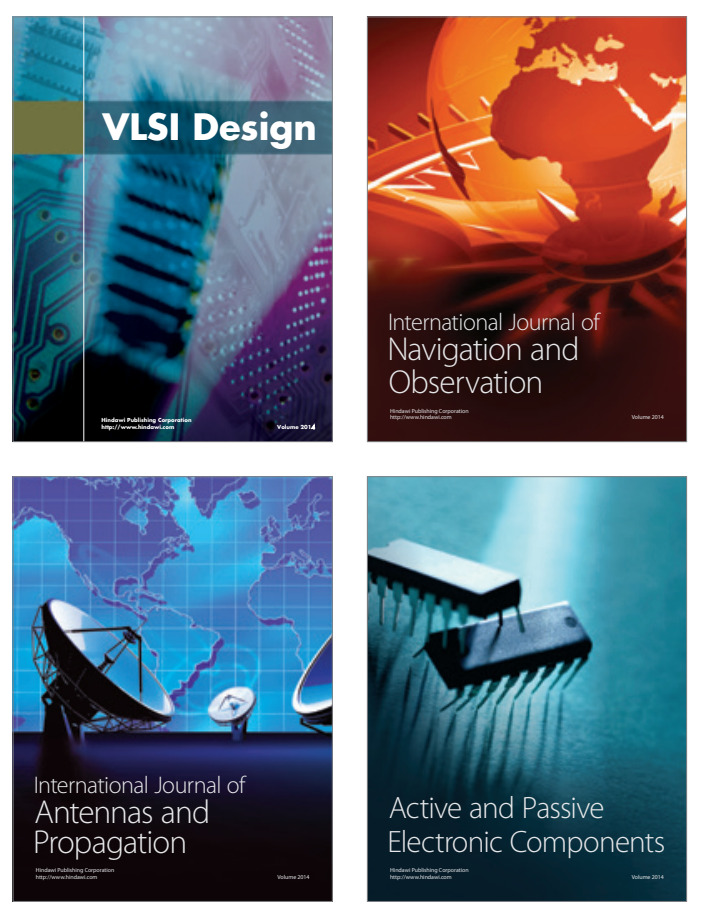
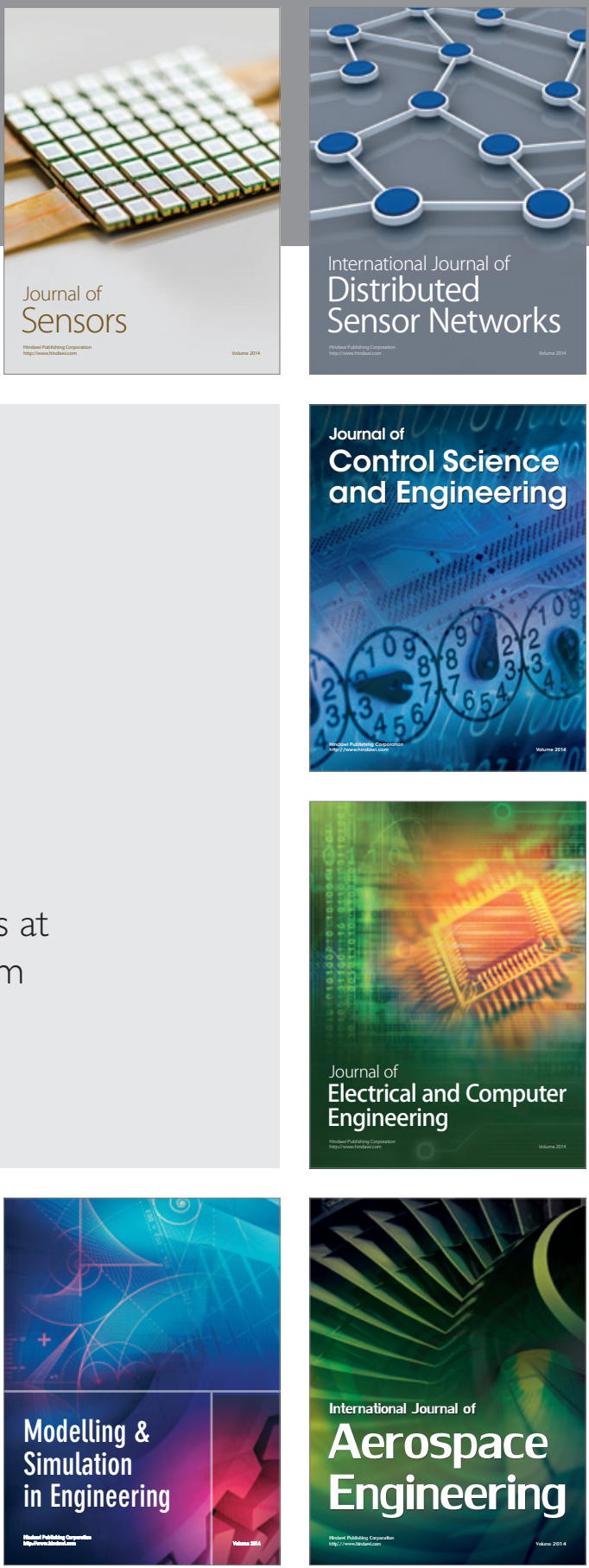

Journal of

Control Science

and Engineering
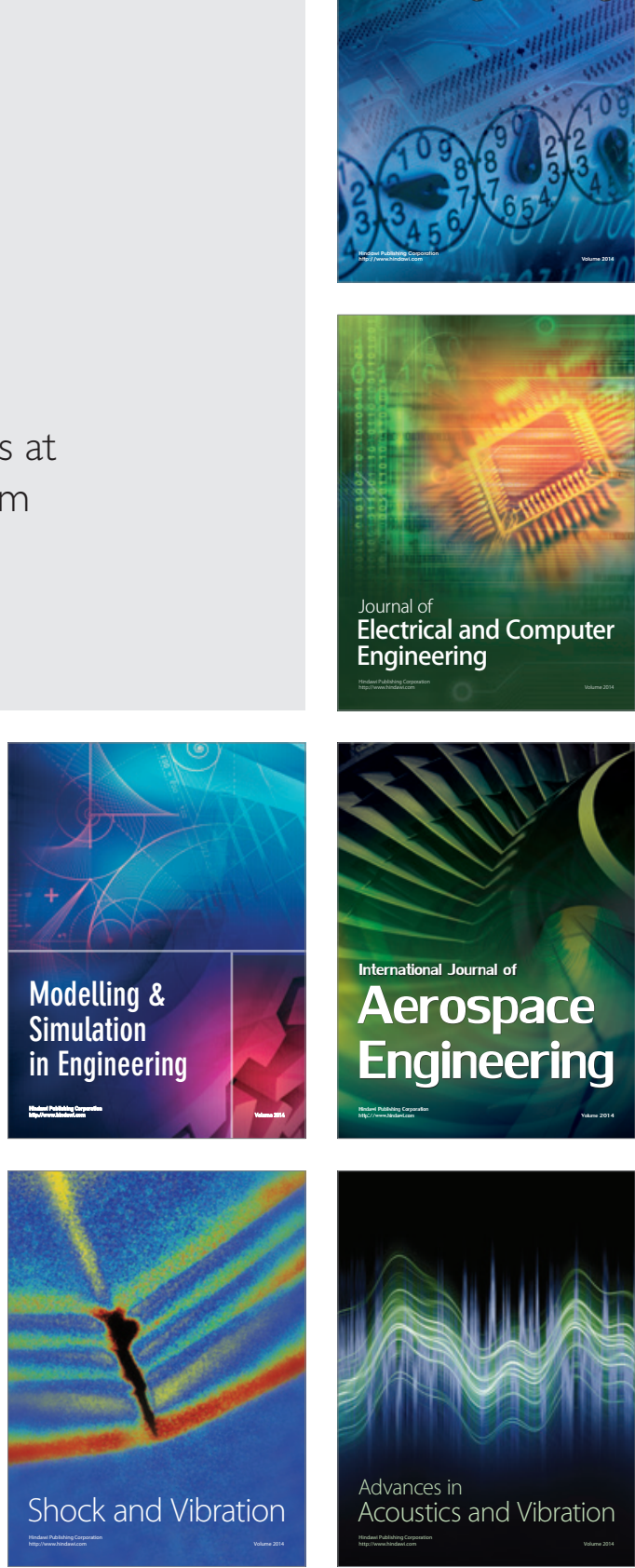